\title{
Stability of the Bloch wall via the Bogomolnyi decomposition in elliptic coordinates
}

\author{
S.R. Woodford* and I.V. Barashenkovt \\ Department of Mathematics and Applied Mathematics, \\ University of Cape Town, Rondebosch 7701, South Africa
}

(Dated: November 19, 2018)

\begin{abstract}
We consider the one-dimensional anisotropic $X Y$ model in the continuum limit. Stability analysis of its Bloch wall solution is hindered by the nondiagonality of the associated linearised operator and the hessian of energy. We circumvent this difficulty by showing that the energy admits a Bogomolnyi bound in elliptic coordinates and that the Bloch wall saturates it - that is, the Bloch wall renders the energy minimum. Our analysis provides a simple but nontrivial application of the BPS (Bogomolnyi - Prasad - Sommerfield) construction in one dimension, where its use is often believed to be limited to reproducing results obtainable by other means.
\end{abstract}

*On leave of absence from Forschungszentrum Jülich. Postal address: Theorie I, Institut für Festkörperforschung, Forschungszentrum Jülich, D-52428 Jülich, Germany; Electronic address: s.woodford@fz-juelich.de

${ }^{\dagger}$ Electronic address: Igor.Barashenkov@uct.ac.za; igor@odette.mth.uct.ac.za 


\section{INTRODUCTION}

Although the Bogomolnyi decomposition is an indispensable tool for the study of higherdimensional field theories [1], it has seldom been used in one dimension. It does allow one to reduce the order and find topological solitons of one-component models such as the sine-Gordon and $\phi^{4}$-theory, but these can be obtained with less effort simply by using an integrating factor. It is of more value for multicomponent [2] and lattice [3] systems, but all models that benefited from its use had to be specifically designed to admit such a decomposition.

In this paper, we apply the Bogomolnyi construction to a system that has been studied for more than forty years: the anisotropic $X Y$ model. It has long been believed that the Bloch-wall solutions of this model are stable; this fact has been demonstrated numerically but never proven analytically. Here, by transforming to elliptic coordinates, we find the Bogomolnyi bound for the energy and show that the Bloch wall minimizes the energy in the corresponding topological sector; this proves its stability. (Note that the Bogomolnyi construction cannot be carried out in the original coordinates.)

An outline of the paper is as follows. In the next section we introduce the (stationary) anisotropic $X Y$ model and its domain-wall solutions. We also describe three different time-dependent extensions of the model which correspond to three different types of behaviour, viz., dissipative, conservative relativistic and conservative nonrelativistic dynamics. In section III we show that because of the nondiagonality of the linearised operator and the second variation of energy, the standard methods are inadequate for proving the stability of the Bloch wall — in each of the three cases. Section [V contains the main result of this paper: the proof of the energy minimization by the Bloch wall. Finally, section $\mathrm{V}$ summarises the results of our work.

\section{THE MODEL}

\section{A. Anisotropic easy-axis ferromagnet near the Curie temperature}

The anisotropic $X Y$ model was originally introduced to describe domain walls in an easyaxis ferromagnet near the Curie point [4, 5]. In the study of domain walls, the magnetization is assumed to vary only in $x$ direction and the magnetization vector $\mathbf{M}$ is taken to lie in the 
$y z$ plane. (With this notation, the system should have been called the $Y Z$ model but we keep the traditional name to avoid confusion.) In the continuum limit, the model is defined by its free energy expansion [4, 5]:

$$
E=\int\left[\frac{1}{2}\left(\partial_{x} \mathbf{M}\right)^{2}-(1+h) \mathbf{M}^{2}+\frac{1}{2} \mathbf{M}^{4}+2 h M_{y}^{2}+\mathcal{E}_{0}\right] d x
$$

The first term in (1) is the exchange energy, which is minimized when $\mathbf{M}=$ const. The combination of the second and third terms makes the $|\mathbf{M}| \neq 0$ ground state energetically

preferable to the state with $|\mathbf{M}|=0$. Anisotropy is caused by the term $2 h M_{y}^{2}$; the parameter $h$ is assumed positive, making the $z$ direction the easy-axis. Finally, the coefficient $(1+h)$ is introduced to simplify the subsequent formulas and a constant $\mathcal{E}_{0}$ has been added to the integrand to ensure that the free energy is finite.

Defining $\psi=M_{y}+i M_{z}$, we can recast the free energy in the form

$$
E=\int\left[\frac{1}{2}\left|\psi_{x}\right|^{2}+\frac{1}{2}|\psi|^{4}-|\psi|^{2}+\frac{h}{2}\left(\psi^{2}+\psi^{* 2}\right)+\mathcal{E}_{0}\right] d x .
$$

This is the form that we shall be working with in this paper.

\section{B. Anisotropic easy-plane ferromagnet in external field}

The same free energy expansion (2) describes one more, unrelated, magnetic system: a weakly anisotropic easy-plane ferromagnet in an external field perpendicular to the easy plane [6]. In terms of the magnetization vector $\mathbf{M}$, the free energy of this system is given by

$$
E=\int\left[\frac{1}{2}\left(\partial_{x} \mathbf{M}\right)^{2}+\frac{\beta}{2} M_{z}^{2}+\frac{\epsilon \beta}{2} M_{x}^{2}-H M_{z}+\mathcal{E}_{0}\right] d x
$$

where $H>0$ is an applied field, the anisotropy parameters $\beta$ and $\epsilon \beta$ are positive, and $\epsilon \ll 1$. In this model, the $x y$ plane is the easy plane, with a weak anisotropy favouring the $y$ direction. Far from the Curie point, the magnetization is hard, i.e. the magnitude of the magnetisation vector is constant: $\mathbf{M}^{2}=M_{0}^{2}=$ const. [For this reason equation (3) has no terms in $\mathbf{M}^{2}$ and $\mathbf{M}^{4}$.]

We assume that the the magnitude of the external magnetic field is close to $\beta M_{0}: H=$ $\beta M_{0}-\epsilon q$, where $q$ is a quantity of order 1 . The (degenerate) ground states will then have the magnetization vector almost parallel to the field:

$$
\mathbf{M}^{( \pm)} \approx\left(0, \pm \sqrt{2 \epsilon q M_{0} / \beta}, M_{0}-\epsilon q / \beta\right)
$$


The deviation from the reference vector $\mathbf{M}=\left(0,0, M_{0}\right)$ pointing in the direction of the field can be characterised using the complex variable $\psi=(\beta / 2 \epsilon s)^{1 / 2}\left(M_{x}-i M_{y}\right)$, where $s \equiv q M_{0}-\beta M_{0}^{2} / 2$. The $M_{z}$ component of the magnetisation is expressible using $\mathbf{M}^{2}=$ $M_{0}^{2}=$ const:

$$
M_{z}=M_{0} \sqrt{1-\frac{2 \epsilon s}{\beta M_{0}^{2}}|\psi|^{2}} .
$$

Transforming to $\tilde{x}=\left(\epsilon s / 2 \alpha M_{0}^{2}\right)^{1 / 2} x$ and keeping terms up to $\epsilon^{2}$, the free energy (3) reduces to equation (2) where $h=\beta M_{0}^{2} / 2 s$ and we have dropped the tilde over $x$. Unlike the anisotropic $X Y$ model considered in the previous subsection, the domain walls in the present system interpolate between the nearly-parallel ground states $\mathbf{M}^{(+)}$and $\mathbf{M}^{(-)}$. It is fitting to note here that due to the relative weakness of magnetic anisotropy, the field $H \approx \beta M_{0}$ will generally be well within the range of modern experiments. For example, the martensite phase of NiMnGa is a weakly anisotropic easy-plane ferromagnet with $\beta \approx 2 \times 10^{-6} \mathrm{Tm} / \mathrm{A}$ and $M_{0} \approx 5 \times 10^{5} \mathrm{~A} / \mathrm{m}[7]$. Therefore, when subjected to a magnetic field $H \approx 1 \mathrm{~T}$, it will be described by equation (2).

Finally, we note that the expansion (11)-(2) arises in yet another magnetic context, viz. a strongly anisotropic ferromagnet with spin $S=1[$ [8] .

\section{Bloch and Ising walls}

The free energy (2) is extremised by solutions of the following stationary equation:

$$
\frac{1}{2} \psi_{x x}-|\psi|^{2} \psi+\psi-h \psi^{*}=0
$$

There are two soliton, or kink, solutions available in literature; each describes an interface between two ferromagnetic domains. One is commonly known as the Ising, or Néel, wall $[4,[5,9,10]$ :

$$
\psi_{I}(x)=i A \tanh (A x)
$$

where $A=\sqrt{1+h}$. The second kink solution has the form

$$
\psi_{B}(x)=i A \tanh (B x) \pm C \operatorname{sech}(B x),
$$

where $B=\sqrt{4 h}$ and $C=\sqrt{1-3 h}[5,11,12]$. This solution is referred to as the Bloch wall. The two signs in front of the real part in (6) distinguish Bloch walls of opposite chiralities.

The Ising wall exists for all positive $h$ whereas the Bloch wall exists only for $h<\frac{1}{3}$. 
The Bloch and Ising wall have the same asymptotic behaviour: $\psi_{B, I}(x) \rightarrow \pm i A$ as $x \rightarrow$ $\pm \infty$. This determines the constant $\mathcal{E}_{0}$ that is added to the integrand in (21) to ensure the finiteness of the free energy: $\mathcal{E}_{0}=A^{4} / 2$. The Bloch and Ising walls have energies

$$
E_{B}=2 B\left(A^{2}-B^{2} / 3\right)
$$

and $E_{I}=\frac{4}{3} A^{3}$, respectively. It is easily verified that the energy of the Ising wall is greater than the energy of the Bloch wall for all $h<\frac{1}{3}$.

In addition to the Bloch and Ising walls, equation (44) has a family of nontopological solitons, available in explicit form and describing Bloch-Ising bound states [13]. These will not be considered here.

\section{Three types of dynamical behaviour}

The dynamics of the domain walls is governed by one of three possible time-dependent extensions of equation (44). In the case when the free energy (2) is used to model the ferromagnet near the Curie point, the evolution of the field $\psi$ is dissipative and governed by the Ginsburg-Landau equation [14]:

$$
\psi_{t}=\psi-|\psi|^{2} \psi+\frac{1}{2} \psi_{x x}-h \psi^{*} .
$$

On the other hand, in the case of the ferromagnet with spin $S=1$, the field $\psi$ satisfies a relativistically-invariant equation [8]

$$
\psi_{t t}-\psi_{x x}-2 \psi+2|\psi|^{2} \psi+2 h \psi^{*}=0 .
$$

Originally, this equation was introduced by Montonen [11] in a different context — as an exactly solvable special case of Rajaraman and Weinberg's bag model [9]. Independently, Sarker, Trullinger and Bishop [12] proposed it as an interesting interpolate between the sine-Gordon and the $\phi^{4}$ theories. Accordingly, the Klein-Gordon equation (9) is commonly known as the Montonen-Sarker-Trullinger-Bishop (MSTB) model.

Finally, the magnetisation vector of an anisotropic easy-plane ferromagnet in an external field satisfies the Landau-Lifshitz equation. The perturbation procedure described in section IIB reduces it [6] to the parametrically driven nonlinear Schrödinger equation,

$$
i \psi_{t}+\frac{1}{2} \psi_{x x}-\psi|\psi|^{2}+\psi=h \psi^{*} .
$$


The stability of the Bloch or Ising wall depends on which of the three equations (8) , (9) or (10) governs the evolution of $\psi$. Consequently, the three cases - the Ginsburg-Landau, the Klein-Gordon and the nonlinear Schrödinger — need to be considered separately.

\section{APPROACHES TO STABILITY}

In this section we describe the standard methods of stability analysis and explain why they all fail in the case of the Bloch wall — for each of the three types of evolution.

\section{A. The Ginsburg-Landau and relativistic dynamics}

We start with the Ginsburg-Landau equation, equation (8), and linearise it about the stationary solution $\psi_{0}(x)$, which can be either the Bloch or Ising wall. Decomposing the small perturbation $\delta \psi(x, t)$ into its real and imaginary parts, $\delta \psi(x, t)=\delta \mathcal{R}(x, t)+i \delta \mathcal{I}(x, t)$, and letting

$$
\delta \mathcal{R}(x, t)=\tilde{\delta \mathcal{R}}(x) e^{\lambda t}, \quad \delta \mathcal{I}(x, t)=\tilde{\delta \mathcal{I}}(x) e^{\lambda t}
$$

yields an eigenvalue problem

$$
-\mathcal{H}\left(\begin{array}{c}
\delta \mathcal{R} \\
\delta \mathcal{I}
\end{array}\right)=\lambda\left(\begin{array}{c}
\delta \mathcal{R} \\
\delta \mathcal{I}
\end{array}\right)
$$

where $\mathcal{H}$ is a self-adjoint operator

$$
\mathcal{H}=\left(\begin{array}{lr}
-\frac{1}{2} \partial_{x}^{2}-1+h+3 \mathcal{R}_{0}^{2}+\mathcal{I}_{0}^{2} & 2 \mathcal{R}_{0} \mathcal{I}_{0} \\
2 \mathcal{R}_{0} \mathcal{I}_{0} & -\frac{1}{2} \partial_{x}^{2}-1-h+\mathcal{R}_{0}^{2}+3 \mathcal{I}_{0}^{2}
\end{array}\right)
$$

In (12), $\mathcal{R}_{0}$ and $\mathcal{I}_{0}$ stand for the real and imaginary part of the stationary solution $\psi_{0}$, respectively. The solution $\psi_{0}$ will be unstable if the operator $-\mathcal{H}$ has at least one positive eigenvalue $\lambda$, and stable otherwise.

In the case of the MSTB model [equation (9)], the linearisation about $\psi_{0}$ produces the same eigenvalue problem (11), with the same operator (12), where one just needs to replace $\lambda$ with $\lambda^{2} / 2$. Here we have the same stability criterion as in the Ginsburg-Landau case: the solution $\psi_{0}$ will be unstable if $-\mathcal{H}$ has at least one positive eigenvalue $\lambda^{2} / 2$.

An alternative approach (which still leads to the same criterion, though) is based on considering the Lyapunov functional (see e.g. [15]). The Ginsburg-Landau equation (8) can 
be written as $\psi_{t}=-\delta E / \delta \psi^{*}$, where $E$ is the functional (2). Hence this functional satisfies

$$
E_{t}=-2 \int\left|\psi_{t}\right|^{2} d x
$$

and so we have $E_{t}<0$ unless $\psi$ is a static solution, $\psi_{t}=0$. Now if we could prove that $E[\psi]>E\left[\psi_{0}\right]$ for all $\psi$ in some neighbourhood of $\psi_{0}$, the functional $E$ would be the Lyapunov functional for this solution and hence $\psi_{0}$ would be proven to be stable.

Here we need to make a standard remark on the translational invariance of equations (8) , (9) and (10). The domain wall centred at the origin has the same energy as the wall centred at any other point $x_{0}$ and therefore can never be an isolated minimum of $E$. However, a mere translation of the wall from the origin to the point $x_{0}$ does not imply instability. Therefore, it is physically reasonable to group all configurations obtained from a given $\psi(x)$ by translations $\psi(x) \rightarrow \psi\left(x-x_{0}\right)$ with $-\infty<x_{0}<\infty$, into equivalence classes. Unlike the wall with any particular $x_{0}$, the equivalence class consisting of all translated walls can be an isolated minimum of the energy — defined on the corresponding quotient manifold. If the perturbations of the wall are assumed to be infinitesimal, the quotient is a linear subspace; namely, it is the quotient of the space of all infinitesimal perturbations of the wall by the subspace spanned by its translation mode $\left(\partial_{x} \mathcal{R}_{0}, \partial_{x} \mathcal{I}_{0}\right)$. This quotient space can be conveniently characterised by the orthogonality constraint

$$
\int\left(\partial_{x} \mathcal{R}_{0}, \partial_{x} \mathcal{I}_{0}\right)\left(\begin{array}{c}
\delta \mathcal{R} \\
\delta \mathcal{I}
\end{array}\right) d x=0 .
$$

In what follows, the subspace of perturbations defined by the constraint (13) will be denoted $\mathcal{S}$.

To check whether $\psi_{0}$ renders $E[\psi]$ a minimum in $\mathcal{S}$, the functional is expanded about the stationary point $\psi_{0}$ :

$$
E[\psi]=E_{0}+\frac{1}{2} \delta^{2} E+\ldots
$$

Here $\psi=\psi_{0}+\delta \mathcal{R}+i \delta \mathcal{I}$, and the second variation has the form

$$
\frac{1}{2} \delta^{2} E=\int(\delta \mathcal{R}, \delta \mathcal{I}) \mathcal{H}\left(\begin{array}{c}
\delta \mathcal{R} \\
\delta \mathcal{I}
\end{array}\right) d x
$$

where the hessian $\mathcal{H}$ coincides with the linearised operator (12). The solution $\psi_{0}$ will minimise $E[\psi]$ in $\mathcal{S}$ provided $\mathcal{H}$ has no negative or zero eigenvalues other than the one associated with the translation mode. 
A Lyapunov functional can also be used in the case of the relativistic dynamics (9) (see e.g. [16]). Here, as a candidate functional one considers the total energy

$$
E_{\text {total }}\left[\psi, \psi_{t}\right]=\frac{1}{2} \int\left|\psi_{t}\right|^{2} d x+E[\psi],
$$

where $E[\psi]$ is as in (2). The energy is conserved, and hence $E_{\text {total }}$ will define the Lyapunov functional for the solution $\psi_{0}$ if it renders this functional a minimum in $\mathcal{S}$. Since the first term in (15) is minimised by any static configuration, it is sufficient to check whether $\psi_{0}(x)$ renders the functional $E[\psi]$ a minimum. Consequently, the minimisation problem reduces to the eigenvalue problem (11).

Thus, we have the same stability criterion in the case of the Ginsburg-Landau and relativistic dynamics - one has to prove that the solution minimises the functional (2) under the constraint (13), or, equivalently, show that the operator $\mathcal{H}$ has no negative or zero eigenvalues other than the translation mode.

In the case of the Ising wall (5), we have $\mathcal{R}_{0}(x)=0$ and the operator (12) is diagonal. Its eigenvalues can be readily found and the above criterion easily implemented. Thus, it was

shown in [8, 9, 17, 18] that the Ising wall is stable for $h>\frac{1}{3}$ and unstable for $h<\frac{1}{3}$. (See [19] for the generalisation to a nonvariational case.)

In the case of the Bloch wall, on the other hand, the operator $\mathcal{H}$ is nondiagonal. This makes it impossible to determine the sign of the lowest eigenvalue of $\mathcal{H}$ using standard analytical methods. Consequently, previous studies had to resort to semi-intuitive and numerical arguments. In particular, it was noted that the energy of the Bloch wall is lower than that of the Ising wall and suggested that the Bloch wall should be stable [11, 12, 20]. This conjecture was supported by results of direct numerical simulations of equation (9) [21] and perturbation theory for small $C(C=\sqrt{1-3 h})$ [8]. However no analytical proof, applicable for all parameter values, has been given so far.

\section{B. The Schrödinger dynamics}

Finally, we discuss the parametrically driven nonlinear Schrödinger equation, equation (10). In this case, the linearisation about $\psi_{0}$ produces a symplectic eigenvalue problem

$$
\mathcal{H}\left(\begin{array}{c}
\delta \mathcal{R} \\
\delta \mathcal{I}
\end{array}\right)=\lambda J\left(\begin{array}{c}
\delta \mathcal{R} \\
\delta \mathcal{I}
\end{array}\right), \quad J=\left(\begin{array}{rr}
0 & -1 \\
1 & 0
\end{array}\right)
$$


where $\mathcal{H}$ is as in (12). The product operator $J^{-1} \mathcal{H}$ is non-self-adjoint and hence its eigenvalues can be complex. The solution $\psi_{0}$ will be unstable if the operator $J^{-1} \mathcal{H}$ has at least one eigenvalue $\lambda$ with positive real part.

The nonlinear Schrödinger equation (10) conserves energy which is given by the integral (2); hence the energy is a potential Lyapunov functional for equation (10). One can therefore try to establish the stability of $\psi_{0}$ by proving that it renders the energy minimum under the constraint (13); this happens when the operator $\mathcal{H}$ in the second variation (14) does not have negative or zero eigenvalues other than the translation mode. Note that the criteria based on the linearisation and the energy minimality appeal to eigenvalues of different operators here, $J^{-1} \mathcal{H}$ and $\mathcal{H}$, respectively. However, it is not difficult to show that the positive definiteness of $\mathcal{H}$ implies that $J^{-1} \mathcal{H}$ does not have eigenvalues with positive real part. We include a proof of this simple fact in the Appendix.

As we have pointed out in the previous subsection, the operator $\mathcal{H}$ associated with the Ising wall is diagonal. Making use of this property, it was proved in [6] that $J^{-1} \mathcal{H}$ does not have eigenvalues with positive real part and the Ising wall is stable for all $h>0$. On the other hand, in the case of the Bloch wall, the operator $\mathcal{H}$ in (11) and (16) is nondiagonal. This prevents the determination of the sign of the lowest eigenvalue of $\mathcal{H}$, or testing the existence of unstable eigenvalues of $J^{-1} \mathcal{H}$, using any of the standard analytical approaches. The eigenvalue problems (11) and (16) can of course be studied numerically; this was done in Ref.[6] where the Bloch wall was found to be stable for all examined values of $h$. However, numerical solutions tend to overlook subtleties (e.g. exponentially small eigenvalues) and give limited insights into the structure of the configuration space. This motivates our search for an analytical stability proof.

We provide such a proof in the next section.

\section{IV. $X Y$ MODEL IN ELLIPTIC COORDINATES}

Returning to the energy (2), we define elliptic coordinates on the $(\operatorname{Re} \psi, \operatorname{Im} \psi)$-plane:

$$
\psi=B(\sinh u \sin v+i \cosh u \cos v) .
$$

Here, $u(x) \geq 0$ and $0 \leq v(x) \leq 2 \pi$ are continuous fields.

The use of elliptic coordinates was pioneered by Trullinger and DeLeonardis who utilised 
these in their calculation of the partition function for the MSTB model [22]. Elliptic coordinates allow the separation of variables in the effective Schrödinger equation that arises in their transfer-matrix approach (see also [20]). Subsequently, Ito [23] used elliptic coordinates to separate variables in the Hamilton-Jacobi formulation of equation (44) (see also [24]).

Transforming to the elliptic coordinates (17), the energy functional (2) acquires the form

$$
E=2 h \int\left[\left(\sinh ^{2} u+\sin ^{2} v\right)\left(u_{x}^{2}+v_{x}^{2}\right)+\frac{f^{2}(u)+g^{2}(v)}{\sinh ^{2} u+\sin ^{2} v}\right] d x,
$$

where

$$
\begin{array}{r}
f(u)=B \sinh u\left(\cosh ^{2} u-\frac{A^{2}}{B^{2}}\right), \\
g(v)=B \sin v\left(\frac{A^{2}}{B^{2}}-\cos ^{2} v\right) .
\end{array}
$$

The integrand in (18) admits a Bogomolnyi-type decomposition

$$
E=2 h \int\left\{\mu(u, v)\left[\left(u_{x}+\frac{f(u)}{\mu(u, v)}\right)^{2}+\left(v_{x}+\frac{g(v)}{\mu(u, v)}\right)^{2}\right]+\Phi_{x}\right\} d x
$$

where

$$
\mu(u, v)=\sinh ^{2} u+\sin ^{2} v \geq 0
$$

and

$$
\Phi_{x}=-2 f(u) u_{x}-2 g(v) v_{x} .
$$

Since both terms in the square brackets in (20) are nonnegative, the energy is bounded from below:

$$
E \geq\left. 2 h \Phi(x)\right|_{-\infty} ^{\infty}
$$

Evaluating the right-hand side of (21) using (19), this inequality is transformed into

$$
E \geq\left.\frac{B}{3}\left\{\left[3 A^{2} \cosh u(x)-B^{2} \cosh ^{3} u(x)\right]+\left[3 A^{2} \cos v(x)-B^{2} \cos ^{3} v(x)\right]\right\}\right|_{-\infty} ^{\infty} .
$$

In terms of the elliptic coordinates, the domain walls' boundary conditions $\psi( \pm \infty)= \pm i \mathrm{~A}$ acquire the form

$$
\begin{array}{ll}
u(-\infty)=\operatorname{arccosh}\left(\frac{A}{B}\right), & v(-\infty)=\pi \\
u(+\infty)=\operatorname{arccosh}\left(\frac{A}{B}\right), & v(+\infty)=0
\end{array}
$$


or

$$
u(+\infty)=\operatorname{arccosh}\left(\frac{A}{B}\right), \quad v(+\infty)=2 \pi .
$$

Using these, the inequality (22) becomes simply

$$
E[\psi] \geq E_{B}
$$

where $E_{B}$ is the energy of the Bloch wall given by (17).

The bound (24) is obviously saturated by the Bloch walls (6) . We now show that, given the boundary conditions (23), the two Bloch walls are the only solutions with the minimum energy. The proof appeals to the Bogomolnyi equations

$$
\begin{gathered}
u_{x}=-\frac{B}{\mu(u, v)} \sinh u\left(\cosh ^{2} u-\frac{A^{2}}{B^{2}}\right), \\
v_{x}=-\frac{B}{\mu(u, v)} \sin v\left(\frac{A^{2}}{B^{2}}-\cos ^{2} v\right),
\end{gathered}
$$

which have to be satisfied by any configuration with $E[\psi]=E_{B}$. In order to prove the uniqueness, it is sufficient to demonstrate that the dynamical system (25) has a unique heteroclinic trajectory connecting the point (23a) to the point (23b) and another unique trajectory connecting (23a) to (23c). To this end, we note that the line $u=\operatorname{arccosh}(A / B)$ is an invariant manifold and that this manifold is attractive: trajectories flow towards this line but no trajectories can leave it. Therefore, the only trajectories connecting the points (23) have to be segments of this straight line. Letting $u=\operatorname{arccosh}(A / B)$, equation (25b) simplifies to

$$
v_{x}=-B \sin v
$$

Subject to the boundary conditions $v(-\infty)=\pi, v(\infty)=0$ and $v(-\infty)=\pi, v(\infty)=2 \pi$, this equation has a unique pair of solutions

$$
\sin v= \pm \operatorname{sech}(B x), \quad \cos v=\tanh (B x) .
$$

(More precisely, these solutions are unique up to translations $x \rightarrow x-x_{0}$.) Inserting equations (26) into (17) yields the right- and left-handed Bloch walls, equation (6)). Consequently, the Bloch walls are indeed the unique minimal energy solutions (modulo translations). This proves their stability — within each of the three evolution equations (8), (9) and (10). 


\section{CONCLUDING REMARKS}

We have utilised the Bogomolnyi construction to prove that the Bloch walls of equation (2) are energy-minimizing kinks. We have also shown that they are unique energy minimizers. Thus for all three evolution equations (8), (9), and (10), the Bloch walls are proven to be stable.

The energy (21) was introduced to describe the anisotropic $X Y$ model. However, the related evolution equations (8), (9) and (10) emerge in several other areas where our results will also be applicable. In particular, the Ginsburg-Landau equation (8) appears as a generic amplitude equation in resonantly forced oscillatory media near the Hopf bifurcation [25]. The Bloch and Ising walls are often regarded as the basic building blocks for the one- and twodimensional patterns arising in such media [26]. Our stability result puts this interpretation on a firmer ground. Next, the MSTB model (9) was studied in the context of quantum field theory [27]. Here, the fact that the model admits a BPS bound is of fundamental importance as it means that it admits a natural supersymmetric extension. Finally, the parametrically driven NLS equation (10) describes Faraday resonance in a wide shallow water channel in the low-viscosity limit [10, 28]. Since the Ising wall has already been shown to be stable

within the NLS equation [6], a similar conclusion obtained now for the Bloch wall reveals an interesting bistability of the two solitons. This bistability should allow experimental realization.

\section{Acknowledgments}

It is a pleasure to thank Alexander Ianovsky and Dmitry Pelinovsky for useful remarks. S.W. was supported by a grant from the Science Faculty of the University of Cape Town. I.B. was supported by the NRF of South Africa under grant 2053723.

APPENDIX A: THE STABILITY-MINIMALITY CORRESPONDENCE FOR THE NONLINEAR SCHRÖDINGER DYNAMICS

While the linearised operator coincides with the hessian of energy in the case of the Ginsburg-Landau and relativistic dynamics, the two operators are different in the nonlinear Schrödinger case. The hessian $\mathcal{H}$ is given by equation (12), whereas the linearised operator 
is $J^{-1} \mathcal{H}$, with $J$ the skew-symmetric matrix (16). The multiplication by a skew-symmetric matrix changes the spectral properties of an operator; for example, the continuous spectrum of $\mathcal{H}$ lies on the positive real axis whereas the continuous spectrum of $J^{-1} \mathcal{H}$ consists of pure imaginary $\lambda$. Therefore it is not obvious how the energy minimality translates into the absence of unstable eigenvalues.

In this appendix we provide a simple proof that the absence of negative and zero eigenvalues of $\left.\mathcal{H}\right|_{\mathcal{S}}$ is sufficient for $J^{-1} \mathcal{H}$ not to have eigenvalues with positive real part. This fact is usually familiar to workers in this field; for the comprehensive treatment, including the necessary conditions for stability and the eigenvalue count, see [29].

First of all, we note that if $\lambda$ is an eigenvalue of the operator $J^{-1} \mathcal{H}$ in (16), associated with an eigenvector

$$
\mathbf{z}(x)=\left(\begin{array}{c}
a(x) \\
b(x)
\end{array}\right),
$$

then $-\lambda$ is also an eigenvalue, associated with the eigenvector

$$
\tilde{\mathbf{z}}(x)=\left(\begin{array}{c}
a(-x) \\
-b(-x)
\end{array}\right) .
$$

[This follows from the fact that both for the Bloch and Ising wall, the off-diagonal elements of the matrix $\mathcal{H}$ in (12) are odd functions of $x: \mathcal{H}_{12}(x)=\mathcal{H}_{21}(x)=-\mathcal{H}_{21}(-x)$.] Hence real eigenvalues always come in $(\lambda,-\lambda)$ pairs. On the other hand, if $\lambda$ is a complex eigenvalue with an eigenvector $\mathbf{z}(x)$, then $\lambda^{*}$ is an eigenvalue with an eigenvector $\mathbf{z}^{*}(x)$. Therefore, complex eigenvalues appear in $\left(\lambda,-\lambda, \lambda^{*},-\lambda^{*}\right)$ quadruplets. Next, if

$$
\mathcal{H} \mathbf{z}=\lambda J \mathbf{z}, \quad \operatorname{Re} \lambda \neq 0,
$$

the eigenvector $\mathbf{z}$ satisfies an identity

$$
\left(\mathbf{z}^{*}, J \mathbf{z}\right)=0
$$

which follows from the self-adjointness of the operator $\mathcal{H}$. In (A2), we used the notation

$$
\left(\mathbf{z}_{1}, \mathbf{z}_{2}\right)=\int\left[a_{1}(x) a_{2}(x)+b_{1}(x) b_{2}(x)\right] d x,
$$

where $a_{i}$ and $b_{i}$ are the (complex) components of the vector $\mathbf{z}_{i}$, i.e.

$$
\mathbf{z}_{i}(x)=\left(\begin{array}{c}
a_{i}(x) \\
b_{i}(x)
\end{array}\right), \quad i=1,2 .
$$


Assume now that the operator $J^{-1} \mathcal{H}$ has an eigenvalue $\lambda$ with $\operatorname{Re} \lambda>0$, with the eigenvector $\mathbf{z}$. It is not difficult to show that the quadratic form (14) calculated on the function

$$
\mathbf{y}=C \mathbf{z}+C^{*} \mathbf{z}^{*}+\tilde{C} \tilde{\mathbf{z}}+\tilde{C}^{*} \tilde{\mathbf{z}}^{*}
$$

where $C$ and $\tilde{C}$ are complex coefficients, is either sign-indefinite, or equals zero. Indeed, substituting (A33) into (14) and making use of (A2), we get

$$
\frac{1}{2} \delta^{2} E[\mathbf{y}]=2 C \tilde{C} \lambda(\tilde{\mathbf{z}}, J \mathbf{z})+\text { c.c. }+C^{*} \tilde{C}\left(\lambda+\lambda^{*}\right)\left(\tilde{\mathbf{z}}, J \mathbf{z}^{*}\right)+\text { c.c. }
$$

where $\tilde{\mathbf{z}}$ is as in (A1) and c.c. stands for the complex conjugate of the immediately preceding term. The expression ( $(\underline{\mathrm{A} 4})$ is either zero or changes its sign under $C \rightarrow-C$. (This conclusion obviously remains valid if the eigenvalue $\lambda$ is real.)

Thus if the form $\delta^{2} E$ is positive definite on the subspace $\mathcal{S}$ defined by the constraint (13) - the condition satisfied if the operator $\mathcal{H}$ does not have negative or zero eigenvalues other than the translation mode - the product operator $J^{-1} \mathcal{H}$ cannot have eigenvalues with positive real part.

[1] R. Rajaraman. Solitons and Instantons. North-Holland, Amsterdam, 1982;

Y. Yang. Solitons in Field Theory and Nonlinear Analysis. Springer-Verlag, New York (2001);

N. Manton and P. Sutcliffe. Topological Solitons. Cambridge University Press, Cambridge (2004);

J. Polchinski. String Theory. Cambridge University Press, Cambridge (2005);

E. J. Weinberg and P. J. Yi, Phys. Rep. 438, 65 (2007)

[2] D. Bazeia, R. F. Ribeiro and M. M. Santos, Phys. Rev. D 54, 1852 (1996);

D. Bazeia, H. Boschi-Filho and F. A. Brito, JHEP 4, 28 (1999);

D. Bazeia and F. A. Brito, Phys. Rev. D 61, 105019 (2000);

D. Bazeia, J. Menezes and M. M. Santos, Phys. Lett. B 521, 418 (2001);

A. Alonso Izquierdo, M.A. González León and J. Mateos Guilarte, Phys. Rev. D 65, 085012 (2002)

[3] J.M. Speight and R.S. Ward, Nonlinearity 7475 (1994)

[4] L. N. Bulaevskii and V. L. Ginzburg, Sov. Phys. JETP 18, 530 (1964) 
[5] J. Lajzerowicz and J. J. Niez, J. Phys. (Paris) 40, L165 (1979)

[6] I. V. Barashenkov, S. R. Woodford and E. V. Zemlyanaya, Phys. Rev. Lett. 90, 054103 (2003)

[7] L. Straka and O. Heczko, J. Appl. Phys. 93, 8636 (2003);

V.A. Chernenko, V.A. Lvov, S. Besseghini and Y. Murakami, Scripta Materialia 55307 (2006)

[8] B.A. Ivanov, A.N. Kichizhiev, and Yu.N. Mitsai. Sov. Phys. JETP 75329 (1992)

[9] R. Rajaraman and E.J. Weinberg, Phys. Rev. D 11, 2950 (1975)

[10] C. Elphick and E. Meron, Phys. Rev. A 403226 (1989)

[11] C. Montonen, Nucl. Phys. B 112, 349 (1976)

[12] S Sarker, S E Trullinger, and A R Bishop, Phys. Lett. A 59, 255 (1976)

[13] I V Barashenkov and S R Woodford, Phys. Rev. E 71026613 (2005);

I V Barashenkov and S R Woodford, Phys. Rev. E 75026605 (2007)

[14] Y. Pomeau, Physica D 51546 (1991);

P. Coullet, J. Lega, and Y. Pomeau, Europhys. Lett. 15221 (1991);

A. Gordon and R. Salditt, Solid State Commun. 82911 (1992)

[15] G. Izús, R. Deza, O. Ramírez, H.S. Wio, D.H. Zanette, C. Borzi, Phys. Rev. A 52129 (1995);

J A Powell, J. Math. Biol. 35729 (1997)

[16] E W Laedke, K H Spatschek and L Stenflo, J. Math. Phys. 242764 (1983);

E W Laedke and K H Spatschek, In: Differential Geometry, Calculus of Variations and Their Applications. Lecture Notes in Pure and Applied Mathematics, vol.100. Editors G M Rassias and T M Rassias. Marcel Dekker, New York 1985, pp. 335-357;

I V Barashenkov, Phys. Rev. Lett. 771193 (1996)

[17] J Lajzerowicz and J J Niez, In: Solitons and Condensed Matter Physics. Proceedings of the Symposium on Nonlinear (Soliton) Structure and Dynamics in Condensed Matter. Oxford, England, June 27-29, 1978. Editors A R Bishop and T Schneider. Springer-Verlag, Berlin 1978, pp.195-198

[18] H. Ito and H. Tasaki, Phys. Lett. A 113179 (1985)

[19] D.V. Skryabin, A. Yulin, D. Michaelis, W.J. Firth, G.-L. Oppo, U. Peschel and F. Lederer, Phys. Rev. E 64, 056618 (2001)

[20] K R Subbaswamy and S E Trullinger, Physica D 2, 379 (1981)

[21] P Hawrylak, K R Subbaswamy and S E Trullinger, Phys. Rev. D 29, 1154 (1984)

[22] S. E. Trullinger and R. M. DeLeonardis, Phys. Rev. B 225522 (1980) 
[23] H. Ito, Phys. Lett. A 112119 (1985)

[24] A Alonso Izquierdo, M A González León and J. Mateos Guilarte, J. Phys. A: Math. Gen. 31 209 (1998)

[25] P. Coullet, J. Lega, B. Houchmanzadeh and J. Lajzerowicz, Phys. Rev. Lett. 65, 1352 (1990);

P. Coullet and K. Emilsson, Physica D 61, 119 (1992);

K. Staliunas, Journ. Mod. Opt. 42, 1261 (1995);

S. Longhi, Opt. Lett. 21, 860 (1996);

L.S. Tsimring and I.S. Aronson, Phys. Rev. Lett. 79, 213 (1997);

V.J. Sánchez-Morcillo, I. Perez-Arjona, F. Silva, G.J. de Valcarcel and E. Roldan, Opt. Lett. 25, 957 (2000);

G.-L. Oppo, A.J. Scroggie and W.J. Firth, Phys. Rev. E 63, 066209 (2001);

H.-K. Park, Phys. Rev. Lett. 86, 1130 (2001);

H.-K. Park and M. Bär, Europhys. Lett. 65, 837 (2004)

[26] B.A. Malomed and A.A. Nepomnyashchy, Europhys. Lett. 27, 649 (1994);

H. Tutu and H. Fujisaka, Phys. Rev. B 509274 (1994);

E. Meron, Discrete Dynamics in Nature and Society 4, 217 (2000);

A. Yochelis, A. Hagberg, E. Meron, A.L. Lin, and H.L. Swinney, SIAM J. Applied Dynamical Systems 1236 (2002);

H. Tutu, Phys. Rev. E 67036112 (2003);

A. Yochelis, C. Elphick, A. Hagberg, E. Meron, Physica D 199201 (2004);

D. Gomila, P. Colet, G.-L. Oppo and M. San Miguel, J. Opt. B: Quantum Semiclass. Opt. 6, S265 (2004);

[27] J. Mateos Guilarte, Ann. Phys. (NY) 188307 (1988)

[28] J.W. Miles, J. Fluid Mech. 148, 451 (1984);

B. Denardo, W. Wright, S. Putterman, and A. Larraza, Phys. Rev. Lett. 64, 1518 (1990);

W. Chen, L. Lu, and Y. Zhu, Phys. Rev. E 71, 036622 (2005)

[29] T. Kapitula, P. G. Kevrekidis, B. Sandstede, Physica D 195263 (2004);

S. Cuccagna, D. Pelinovsky and V. Vougalter, Communications in Pure and Applied Mathematics, 581 (2005);

T. Kapitula, P. G. Kevrekidis, B. Sandstede, Physica D 201199 (2005);

D. E. Pelinovsky, Proc. Roy. Soc. Lond. A 461783 (2005); 
D. E. Pelinovsky and P. G. Kevrekidis, To appear in: Z. angew. Math. Phys. (2008) 